\title{
Lipid recovery from a vegetable oil emulsion using microbial enrichment cultures
}

\author{
Jelmer Tamis ${ }^{1 *}$, Dimitry Y Sorokin ${ }^{1,2}$, Yang Jiang $^{1}$, Mark C M van Loosdrecht ${ }^{1}$ and Robbert Kleerebezem ${ }^{1}$
}

\begin{abstract}
Background: Many waste streams have a relatively high vegetable oil content, which is a potential resource that should be recovered. Microbial storage compound production for the recovery of lipids from lipid-water emulsions with open (unsterilized) microbial cultures was investigated in a sequencing batch reactor using a diluted vegetable oil emulsion as model substrate.

Results: After feeding, triacylglycerides (TAG) were accumulated intracellular by the microbial enrichment culture and subsequently used for growth in the remainder of the sequencing batch cycle. Roughly $50 \%$ of the added TAG could be recovered as intracellular lipids in this culture. The maximum lipid storage capacity of the enrichment culture was $54 \%$ on volatile suspended solids (VSS) mass basis in a separate fed-batch accumulation experiment. The microbial community was dominated by a lipolytic fungus, Trichosporon gracile, that was responsible for intracellular lipid accumulation but also a significant fraction of lipolytic and long chain fatty-acid-utilizing bacteria was present.
\end{abstract}

Conclusion: Herewith, we demonstrate an effective strategy for enrichment of a microbial community that can accumulate significant amounts of lipids from wastewaters without the need for sterilization of substrates or equipment. Further optimization of this process will make recovery of lipids from wastewater possible.

Keywords: Vegetable oil, Storage compounds, Microbial enrichment culture, Feast-famine, Resource recovery

\section{Introduction}

The use of agro-industrial organic residues for the production of valuable commodities is a logical step towards a bio-based economy. Traditionally, these wastes are used for biogas or compost production. Recently, several alternatives for the production of more valuable compounds have been proposed, for example, the production of biopolymers [1,2], volatile fatty acids [3], or medium chain length fatty acids [4]. One of the critical issues related to resource recovery from wastewater is the efficient up-concentration and purification of the valuable compounds that are present in diluted form in the wastewater. In general, the production of storage compounds with microbial cultures from diluted waste streams improves the efficiency of resource recovery since the product is concentrated inside the biomass

\footnotetext{
*Correspondence: j.tamis@tudelft.nl

'Department of Biotechnology, Delft University of Technology, Julianalaan 67, 2628 BC Delft, The Netherlands

Full list of author information is available at the end of the article
}

and can be readily separated from the water using standard sludge settling/separation methods.

A relatively well studied example of a process based on storage compound production by microbial enrichment cultures is the production of polyhydroxyalkanoate (PHA) and contents up to $90 \%$ on VSS mass basis have been obtained by application of selective pressure in the form of feast-famine conditions [5,6]. This process is currently further evaluated in industrial environments [7-9]. Interestingly, it has been found that different carbon sources in a feast-famine process can result in formation of different storage compounds: microbial cultures enriched on glucose or starch, produced polyglucose as storage compound [10,11], a microbial culture enriched on glycerol produced a mixture of polyglucose and PHA [12], microbial communities enriched on different types of volatile fatty acids (VFA) produced different varieties of PHA [13,14], and a microbial culture enriched on methanol did not produce any storage compounds [15]. While the above examples illustrate the many substrate 
types that have been investigated, the fate of lipids as substrate in a feast-famine process remains unclear.

Lipids are an important constituent of many types of wastewater, especially triacylglycerides (TAG), present in (among others) effluents from vegetable oil crop processing industries. For example, in the wastewater from the palm oil industry, high concentrations of emulsified oil are found that are hard to recover using physical-chemical methods [16]. Palm oil is a fast growing market with a current production of around 50 Mton/year [17] accompanied by the production of $3 \mathrm{~m}^{3}$ wastewater per ton oil palm oil produced [18] resulting in an estimated palm oil mill effluent flow of 150 million $\mathrm{m}^{3} /$ year.

It was proposed that lipids found in wastewater may be recovered through the use of lipid-accumulating microorganism for the production of biodiesel [19]. The economic feasibility of lipid extraction for diesel production from sludge will depend on the lipid content accumulated in the sludge but a cost of around 1 US \$/1 was reported from sludge containing $10 \%$ lipids [20]. In this approach, the recovery of lipids from wastewater requires a microorganism with an as high TAG storage capacity as possible. However, not all microorganisms store TAG from wastewater in an appropriate way for biodiesel production.

A variety of metabolic pathways related to the microbial conversion of TAG have been described in literature (Figure 1). Due to the relatively large molecule size $\left(\mathrm{M}_{\mathrm{w}}\right.$ around 800 to $\left.1,000 \mathrm{~g} / \mathrm{mol}\right)$ and strongly hydrophobic nature of TAG, the uptake mechanism involves an extracellular hydrolysis step yielding long chain fatty acids (LCFA) and glycerol, which are subsequently transported into the cell [21]. Once the substrate is taken up, different routes are possible for the production of storage compounds: for example, PHA production by pure cultures of prokaryotic strains was reported [22-25] as well as the intracellular storage of TAG by pure cultures, for example, by oleaginous yeasts [26,27] or bacteria [28,29].

One approach for the recovery of lipids from wastewater is the cultivation of pure cultures of strains with very high lipid storage capacity. This approach has been evaluated in laboratory experiments with synthetic substrates and sterilized molasses [27]. However, since sterilization of diluted wastewater streams for biodiesel production is likely economically unfeasible, the use of unsterilized wastewater was evaluated [30,31]. In these cases, a precultivated pure culture of oleaginous yeast was used to inoculate a batch experiment with acidic wastewater of a distillery as substrate. It was observed that a biomass with a relatively high lipid content (respectively $44 \%$ and $14 \%$ ) could be grown by this method.

A different strategy was proposed by Santamauro et al. [32] in which an oleaginous yeast could be selectively enriched in a low $\mathrm{pH}$ environment. In general, oleaginous yeasts thrive relatively well in low $\mathrm{pH}$ environments. Nevertheless, many non-oleaginous microorganisms are known to be able to grow under low pH conditions [33]. The selective pressure imposed by the low $\mathrm{pH}$ (or temperature) does not intrinsically provide a competitive

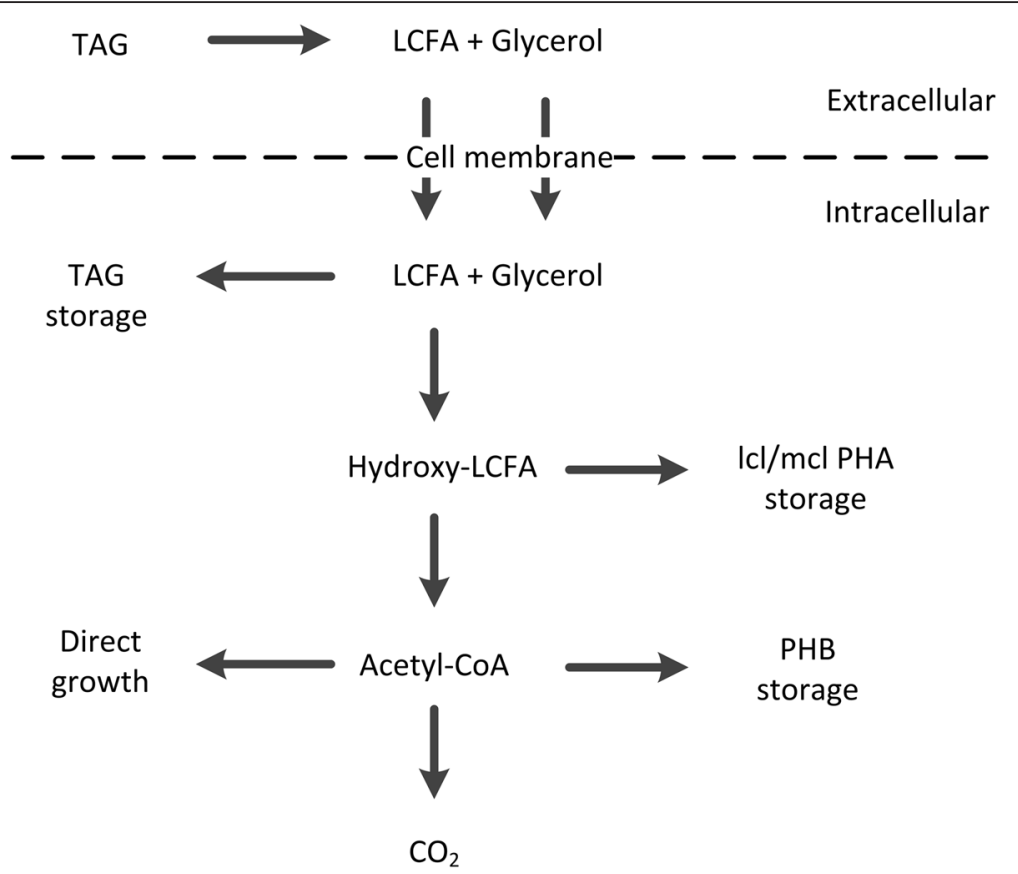

Figure 1 Simplified metabolic pathways of storage compound formation and growth on TAG based on literature reports [21-29,38]. LCFA, long chain fatty acid; PHB, polyhydroxybutyrate; TAG, triacylglycerides. 
advantage for lipid-accumulating microorganisms, which makes the enrichment culture unstable and likely not optimal for lipid accumulation [34].

Since lipid accumulation has been reported as a microbial survival strategy to balance carbon and energy requirements in periods of absence of external substrate [35], in this study, we propose the application of a feastfamine strategy as a novel approach for the enrichment of lipid-accumulating organisms. This strategy is analogous to the feast-famine process for PHA production [6] and is based on ecological principles that provide an intrinsic competitive advantage to lipid-accumulating species. This process potentially enables the recovery of lipids from a broader spectrum of wastewaters, can be operated as sequenced batch or continuously, and alleviates the need for single batch operations with pre-cultivated pure culture inocula. An open (non-sterilized) reactor system was inoculated with wastewater sludge and pulse-fed with a model substrate (soybean oil) to evaluate the potential of the feast-famine principle for the recovery of lipids from diluted lipid-containing emulsions.

\section{Results}

\section{Reactor operation}

After inoculation of the sequencing batch reactor (SBR) with activated sludge (WWTP Dokhaven, The Netherlands) a typical feast-famine pattern became apparent in less than 5 days ( 10 cycles). The length of the feast phase (that is, time it took to take up the lipid substrate added with the influent) was initially more than $3 \mathrm{~h}$ and became more stable after 25 days ( 50 cycles) with a feast phase length of around 1 to $2 \mathrm{~h}$ (Figure 2). Variations in the length of the feast phase are due to the formation of biofilm in the reactor, which had to be periodically removed.
After the reactor had reached a stable operational performance, the carbon mass balance over the system was evaluated by analysis of the influent and effluent in the period of day 68 until day 76 of operation (16 cycles). The average effluent total suspended solids (TSS) concentration was $1.0 \pm 0.16 \mathrm{~g} / \mathrm{l}$ (standard deviation over the dataset of 16 cycles) with an ash fraction of $0.14 \pm 0.03$, resulting in a volatile suspended solids (VSS) concentration of 0.86 $\pm 0.15 \mathrm{~g} / \mathrm{l}$. Additionally, the amount of biofilm that was formed on the reactor wall was removed and measured, indicating an average biofilm growth of 0.25 gVSS per cycle. TOC measurements indicated that the carbon content of the organic solids in the effluent was $0.59( \pm 0.03)$ $\mathrm{gC} / \mathrm{gVSS}$ resulting in a total organic solids production equivalent to $59 \pm 10 \mathrm{Cmmol}$ per cycle. Off-gas measurements showed a $\mathrm{CO}_{2}$ production of $17 \pm 3 \mathrm{mmol}$ per cycle. Herewith, most of the carbon present in the influent substrate $(83 \mathrm{Cmmol} / \mathrm{cycle})$ could be accounted for by carbon in the produced organic solids and $\mathrm{CO}_{2}$ $(76 \pm 10 \mathrm{Cmmol} / \mathrm{cycle})$. The $10 \%$ deviation in the balance is partly due to the complications arising from the reactor wall growth.

To get further insight into the conversions during the cycle, three experiments were performed. For all three cycle experiments, the biomass concentrations and length of feast phase were within the range determined during the steady operational period. In Figure 3, profiles from a typical cycle are shown. For practical purposes and in order to establish the substrate amount accurately, the substrate in these experiments was dosed manually in one short pulse, and the amount of substrate was evaluated by the weight of the dosing syringe before and after addition of the oil.

After dosing the vegetable oil, the reactor liquid became opaque, indicating that the mixture of microorganisms

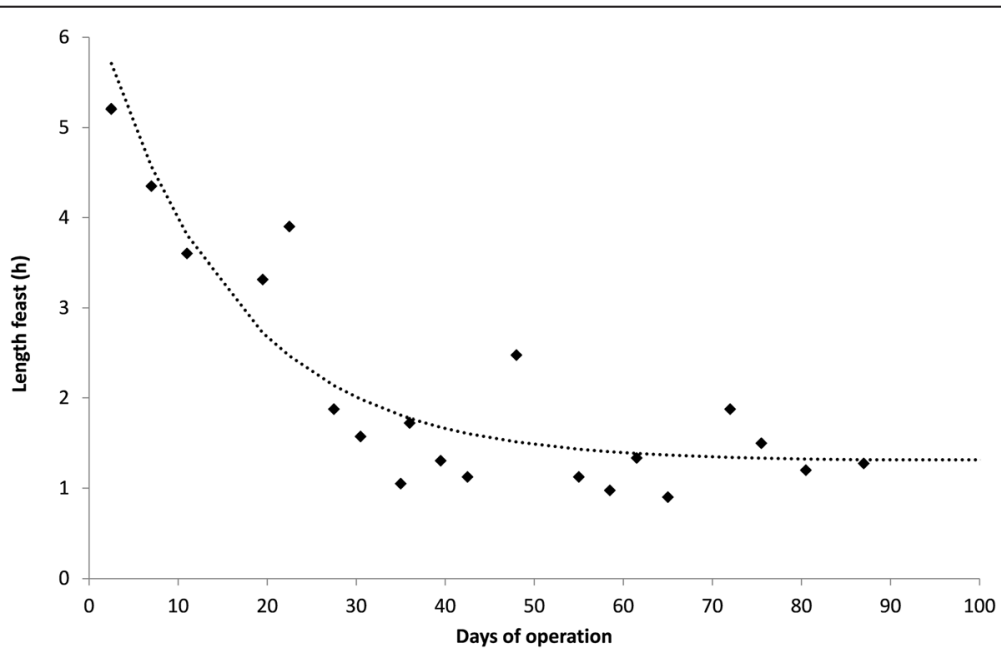

Figure 2 Monitoring of the start-up of the sequencing batch reactor by the evolution of the length of the period needed for TAG substrate uptake (feast phase) by the enrichment culture in the reactor. $h$, hours. 


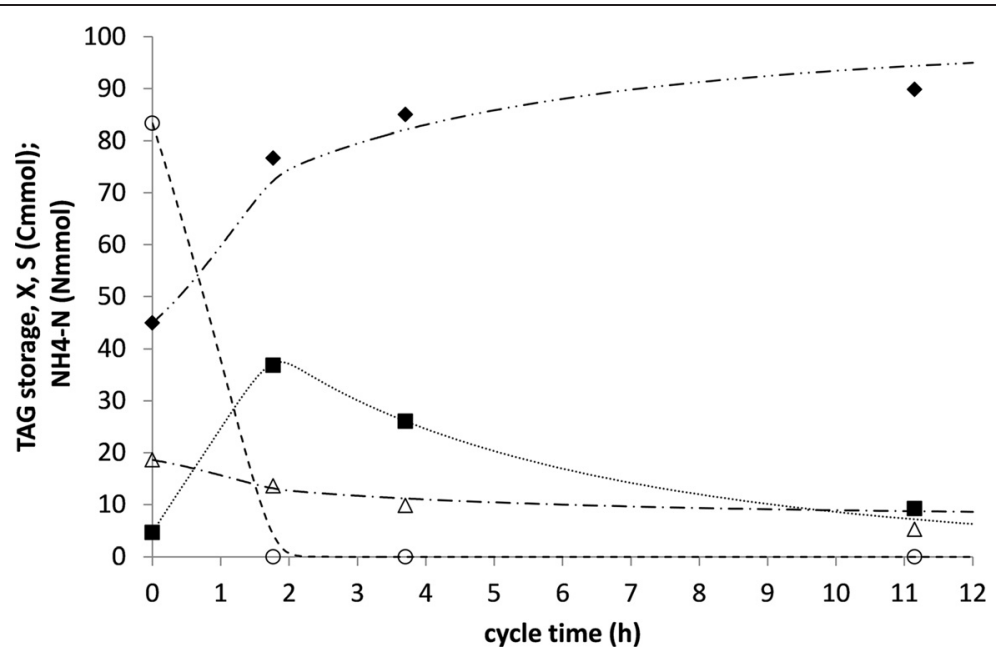

Figure 3 Amounts of TAG substrate $(\circ,----)$, lipid storage $(\mathbf{\bullet}, \cdots \cdot \cdot \cdot)$, active biomass $\left(\bullet,-\cdot-{ }_{-} \cdot--\right)$, and ammonium $\left(\Delta_{1},-\cdot-\cdot-\right)$ during a cycle in the TAG-fed sequencing batch reactor. Symbols represent measurement and lines represent model-based calculations. $h$, hours; TAG, triacylglycerides.

and vegetable oils formed an emulsion; this opaque feature disappeared again at the end of the feast phase (after $2 \mathrm{~h}$ ) when the TAG was depleted from the medium. Gas chromatography (GC) analysis showed that the lipid content of the biomass increased from $6 \% \pm 0.6 \%$ on VSS mass basis, at the start of the cycle, to $25 \% \pm 1.5 \%$ on VSS mass basis at the end of the feast phase (average \pm standard deviation over three cycle experiments), indicating significant storage of lipids (this was also confirmed by microscope images: Figure 4, right). The composition of the accumulated microbial lipids was almost identical to the substrate oil (Table 1), indicating direct utilization of the externally hydrolyzed LCFA and glycerol without their de novo synthesis or conversion. GC analysis showed only peaks that corresponded with the LCFA standard, and no significant peaks corresponding with the PHA standards, suggesting that TAG was the only relevant storage compound present in the culture at any time during the cycle. The lipid content of the cells decreased during the famine phase indicating growth on the stored lipids. Nitrogen was present throughout the cycle, and it was observed that nitrogen was taken up both during the feast and the famine phase, indicating significant microbial growth during the whole SBR cycle. The increase in VSS concentration in the feast phase was higher than that would be expected from TAG storage only, supporting the indication of growth of microorganisms in both the feast phase and the famine phase. A model was calibrated to the experimental data to identify characteristic process parameters (Additional file 1: Appendix C). It was estimated that about half of the substrate was used for growth while the other half was accumulated as intracellular lipids during the feast phase. An overall biomass-specific uptake rate of $1.0 \mathrm{Cmol} /(\mathrm{Cmol} \mathrm{h})$, a yield of lipid storage on substrate of $0.9 \mathrm{Cmol} / \mathrm{Cmol}$ and biomass on lipids of 0.6 to $0.7 \mathrm{Cmol} / \mathrm{Cmol}$ were estimated from the cycle measurements. A summary of the experimental data obtained during steady operation and cycle measurements

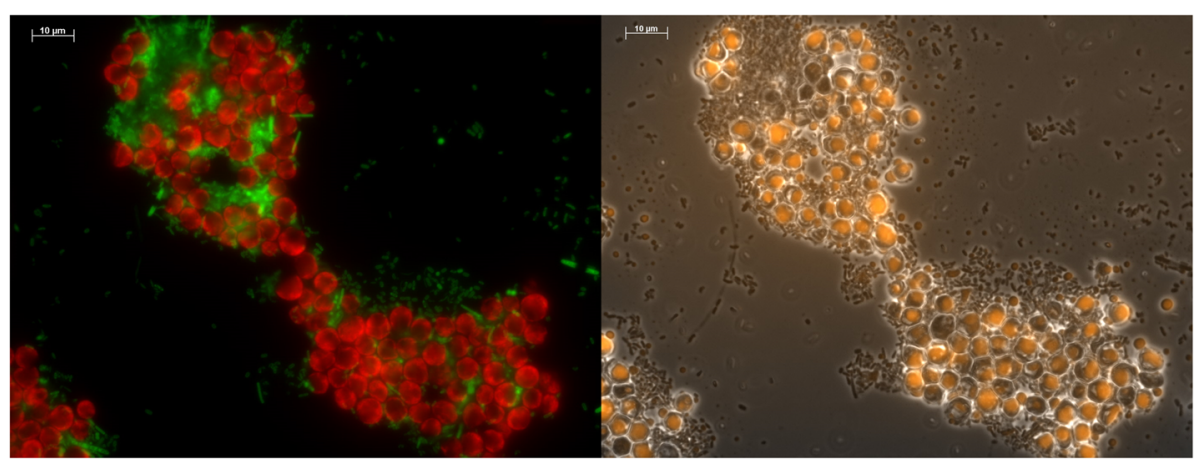

Figure 4 Microscope images of a sample from the reactor at the end of the feast phase. Left: FISH staining with the EUK516 probe to stain eukaryotes (red) and the EUB338 probe to stain prokaryotes (green). Right: Nile blue A staining to identify lipids (orange). 
Table 1 Comparison of the LCFA constituents from literature, used substrate and intracellular storage compounds

\begin{tabular}{llll}
\hline & $\begin{array}{c}\text { Soybean oil [47] } \\
\end{array}$ & $\begin{array}{c}\text { Soybean oil } \\
\text { (this study) }\end{array}$ & $\begin{array}{l}\text { Intracellular } \\
\text { storage (this study) }\end{array}$ \\
\hline Oleic acid & $23 \%$ & $23 \%$ & $25 \% \pm 5 \%$ \\
Linoleic & $53 \%$ & $55 \%$ & $50 \% \pm 6 \%$ \\
a-Linolenic & $8 \%$ & $6 \%$ & $7 \% \pm 2 \%$ \\
Palmitic & $11 \%$ & $12 \%$ & $11 \% \pm 3 \%$ \\
Stearic & $4 \%$ & $4 \%$ & $4 \% \pm 2 \%$
\end{tabular}

For the composition of the intracellular storage, the average and the standard deviation over a dataset of six individual samples are provided. LCFA, long chain fatty acid.

in terms of carbon flows is provided in the (Additional file 1: Appendix B).

The maximum lipid storage capacity of the culture was evaluated using fed-batch experiments in which soybean oil was available in excess. The maximum lipid content appeared to be dependent on the availability of nitrogen. In the absence of a nitrogen source, only $24 \%$ lipid storage on VSS basis was observed while in another experiment with ammonium present in the reactor liquid (C:N molar ratio was 4/1 Cmol/Nmol), a lipid accumulation of $54 \%$ on VSS basis was observed. While the initial lipid accumulation was relatively fast, the lipid content of the culture stabilized after 4 to $5 \mathrm{~h}$ of operation, indicating the maximum storage capacity of the culture under these conditions was reached (Figure 5).

\section{Lipase activity assays}

The lipase activity was measured using a standard test with 4-nitrophenyl-palmitate [36] during the feast and the famine phases. An activity of $0.80 \pm 0.28 \mathrm{mmol} /(1 \mathrm{~min})$ was measured during the feast phase (average \pm standard

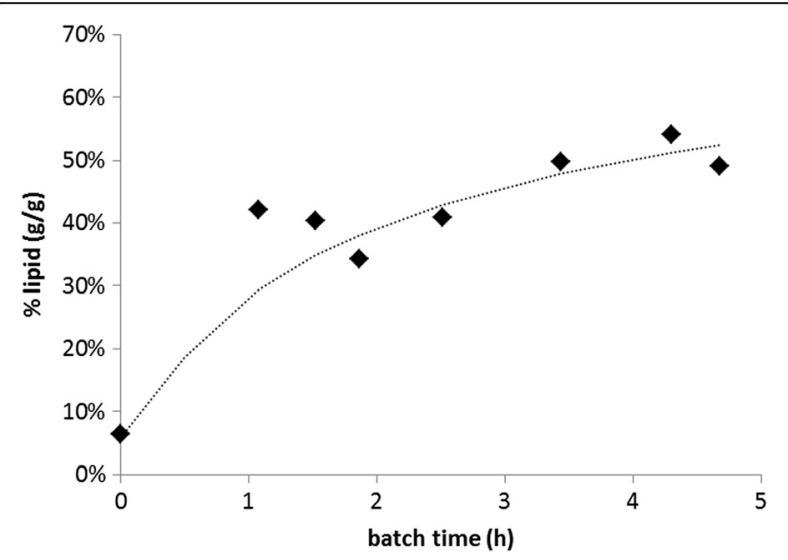

Figure $\mathbf{5}$ The evolution of lipid content of the enriched microbial culture over time in a fed-batch experiment with nutrients present. h, hours. deviation over a dataset of six measurements). During the famine phase, an activity of $0.73 \pm 0.27 \mathrm{mmol} /(\mathrm{l} \mathrm{min})$ was measured (average \pm standard deviation over a dataset of three measurements). This indicated that there was no significant difference in lipase activity between the feast and the famine phases. For all cases, we evaluated the activity of both the reactor broth (with biomass) and the reactor supernatant. The average activity measured in the reactor broth was $0.99 \pm 0.18 \mathrm{mmol} /(1 \mathrm{~min})$ and in the supernatant $0.57 \pm 0.14 \mathrm{mmol} /(\mathrm{lmin})$. This indicated that a significant part of the enzymatic activity was due to extracellular enzymes but also that part of the lipase activity was cell-bound. The measured lipase activity was relatively high compared to the lipid uptake rate observed during the feast phase. Using an uptake of $83 \mathrm{Cmmol}$ (approximately $4 \mathrm{mmol}$ ester bonds) in $2 \mathrm{~h}$ and a reactor volume of $2 \mathrm{l}$, the minimal required lipase activity would be 0.01 to $0.02 \mathrm{mmol} /(\mathrm{lmin})$. This indicated that extracellular lipase activity was not limiting the conversion rates.

\section{Microbial community structure}

The molecular and microscopic analyses of the reactor biomass showed a presence of a dimorphic fungus and several bacterial morphotypes. The direct microscopy results were confirmed by fluorescence in situ hybridization (FISH) analysis which indicated the presence of both eukaryotes and prokaryotes (Figure 4, left). Furthermore, lipid-specific Nile blue A staining indicated that the fungus was involved in lipid storage while the majority of the prokaryotes in the reactor did not contain significant amounts of lipid-like intracellular storage compounds (Figure 4, right).

Denaturing gradient gel electrophoresis (DGGE) was used to characterize the communities. Eukaryotic primerbased PCR products indicated the presence of Trichosporon gracile $(100 \%$ similarity) from a genus known to include several oleaginous species [27,37]. The bacterial-specific DGGE analysis of the reactor biomass indicated the presence of a few proteobacterial phylotypes closely related to the Acetinobacter and Gordonia genera (Figure 6), which are both known to possess metabolic pathways for degradation of hydrophobic substrates $[38,39]$.

Cultivation efforts have been made to try to resolve the role of the microbial species present in the reactor biomass in lipid degradation and utilization of the products. Incubations, with and without antibiotics, allowed the isolation of the eukaryote Trichosporon and four prokaryote microorganisms. The eukaryotic dimorphic fungus $T$. gracile was isolated on lipid plates with antibiotics; it formed mycelium-containing colonies surrounded by zones of lipid hydrolysis (Figure 7). This organism was most versatile in its metabolism, being able to degrade the TAG, utilize the products of hydrolysis (LCFA and glycerol), and reform the TAG inside the cells. The bacterial 


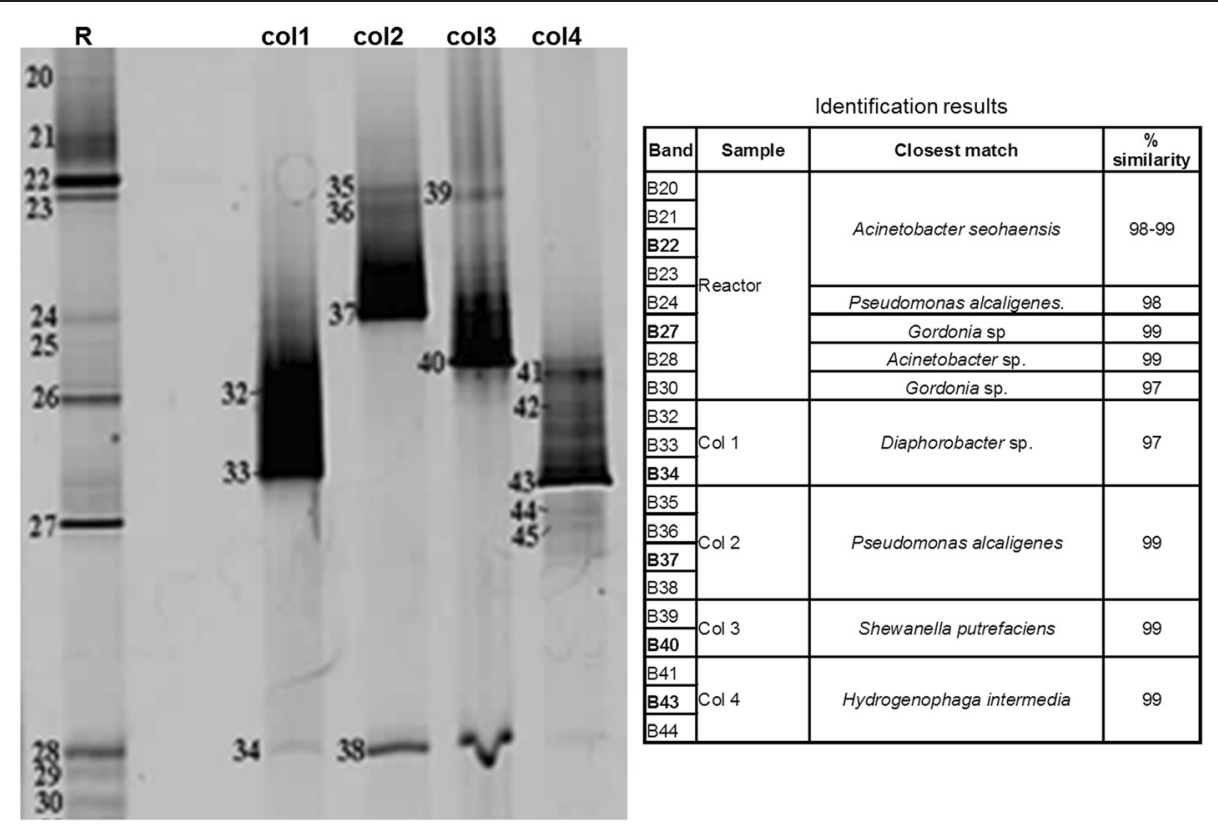

Figure 6 Bacterial-specific DGGE analysis of the reactor biomass and isolates. Col 1, LCFA isolate, long rods; Col 2, lipolytic, short motile rods; Col 3, LCFA isolate, small rods, Col 4: glycerol enrichments; DGGE, denaturing gradient gel electrophoresis; LCFA, long chain fatty acid; $R$, reactor biomass.

enrichments with three different substrates - lipid, LCFA, and glycerol, yielded four isolates: (i) a small motile rodshaped bacterium able to grow with lipid and LCFA with strong lipolytic activity (col2, identified as a Pseudomonas alcaligenes from the betaproteobacteria). Isolates (ii) and (iii) are two different types of LCFA utilizers. The first LCFA degrader (col1) is a long rod with the ability to store lipids inside the cells. This microorganism was identified as a representative of the genus Diaphorobacter from the betaproteobacterial lineage. Although the genus Diaphorobacter is quite common for activated sludge, our isolate is very different in cell morphology from the three species described of this genus. The ability to utilize LCFA has never been tested for this genus. The second LCFA degrader is a small rod with no apparent storage polymers (col3) identified as a representative of the genus Shewanella in the gammaproteobacteria. (iv) Glycerol-specific enrichment resulted in a domination of Hydrogenophaga sp. (betaproteobacterium) represented by small motile rods. Summarizing, the cultivation results showed two functional parts - a eukaryotic dimorphic fungus, doing a complete job of lipid degradation and lipid accumulation, and a bacterial block, basically doing equivalent work but divided between different species (Figure 7).

Despite that two of the bacterial species (Diaphorobacter and Pseudomonas) were clearly recognizable by microscopic inspection of the reactor biomass, only the latter was detected directly by DGGE analysis of the reactor biomass. One of the possible explanations is a DNA extraction bias in the presence of lipids.

\section{Discussion}

A microbial culture capable of accumulating lipids from a dilute emulsion was enriched in a sequencing batch reactor with a feast-famine regime. The development of this enrichment from an activated sludge inoculum occurred relatively fast (25 days). A lipid-storing fungus and a variety of proteobacterial species co-existed in the enrichment culture. Microscopic observation of the reactor biomass indicated that the bacterial phenotypes (except a small population of large cells identified as a Diaphorobacter sp.) did not store significant amounts of lipids but instead used lipids or its immediate hydrolysis products directly as growth substrates.

Analysis of the microbial community by DGGE indicated the presence of the eukaryotic fungus T. gracile, which could be readily isolated by microbial plating techniques with antibiotics and appeared to be able to accumulate significant amount of lipids intracellularly. The bacterial population analysis was more complicated since Gordonia and Acinetobacter species that were detected by DGGE could not be isolated using microbial plating techniques. Gordonia and Acinetobacter species are important species in the lipid-degrading populations that cause foaming in wastewater treatment plants [40], and especially Gordonia species are known to accumulate large amounts of intracellular lipids [41]. Furthermore, while Diaphorobacter was observed in the biomass (based on its morphology) and could be readily isolated by microbial plating; it could not be detected by DGGE analysis. This shows that microbial analysis tools such as microbial 

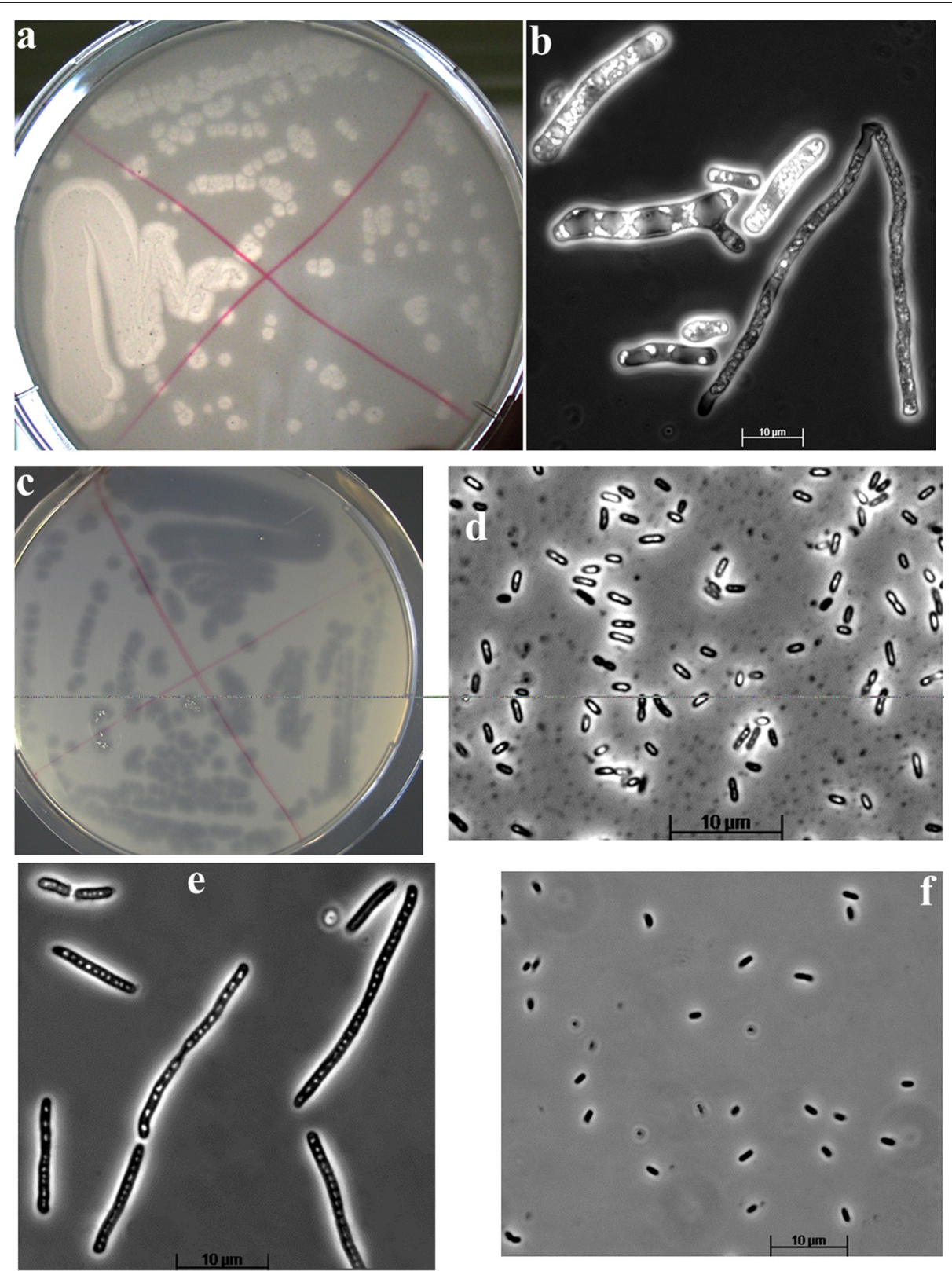

Figure 7 Different functionalities from isolates obtained by plating of the reactor culture. Upper panel: Trichosporon gracile ((a) lypolytic colonies, (b) lipid-storing hyphal cells); middle panel: lipolytic Pseudomonas alcaligenes (col2) ((c) lipolytic colonies, (d) cells in liquid culture with lipid); bottom panel: LCFA-utilizing bacterial isolates ((e) Diaphorobacter sp. (col1) and (f) Hydrogenophaga sp. (col3)).

plating and DGGE can easily introduce bias in the population analysis of a reactor. In conclusion, we could identify and culture the dominant microorganism in the culture, a yeast, but the major bacteria could not be exclusively identified by DGGE or microbial plating.

The presence of the bacterial side population in the reactor may be partly explained by the role of bacteria that by themselves were not able to hydrolyse and use TAG as substrate, but could thrive in the reactor by using the LCFA or glycerol that was liberated through the extracellular lipase activity facilitated by, for example, Trichosporon. Furthermore, the coexistence of storing and non-storing microbes indicates that lipid uptake and storage by $T$. gracile was not fast enough to outcompete direct growth by bacterial lipolytic and LCFA utilizers. The specific uptake rate of the microbial culture was with $1 \mathrm{Cmol} /$ (Cmol h) relatively low compared to similar feast-famine systems on other substrates, for example, an uptake rate of around $4 \mathrm{Cmol} /(\mathrm{Cmol} \mathrm{h})$ was reported for a feast-famine culture cultivated using acetate as carbon source [42]. 
The measured hydrolytic activity was more than an order of magnitude higher than the observed uptake rate suggesting that hydrolysis was not the rate limiting step. It should be noted that the values obtained from the enzyme assay only serve as rough indication since the lipase activity may vary when TAG is the substrate instead of 4-nitrophenol-palmitate $[36,43]$. Factors that potentially limit the lipase activity include transport limitations due to formation of an emulsion during the feast phase [44].

In this study, we show the use of a feast-famine based enrichment strategy for the enrichment of a mixed microbial culture with enhanced lipid accumulation capacity. Remarkably, the maximum accumulation of intracellular lipids of $54 \%$ on VSS basis was only achieved when excess nutrients were supplied. This is in contrast with PHA-producing enrichment cultures, which were reported to achieve higher storage contents during nitrogendeficient fed-batch experiments [45]. Possibly, the major lipid-storing species could not function under nitrogenlimited conditions. Further research may include investigation of the behavior of pure cultures of $T$. gracile to evaluate this hypothesis.

The lipid content of the microbial enrichment culture obtained in this study was around 50\% on VSS mass basis and was mainly related to the presence of the oligeanous yeast $T$. gracile. The Trichosporon genus is known to be able to accumulate high amount of lipids, for example, TAG contents higher than $60 \%$ were reported in pure culture experiments [27]. The high TAG content of these cultures suggest that further improvement may be achieved by development of strategies that provide a competitive advantage for Trichosporon over the bacterial side population.

Nevertheless, the achieved lipid content of around $50 \%$ is already significantly more than the $10 \%$ lipid content in sludge that was used as reference content to evaluate the feasibility biodiesel production [20] or and even more than the oil content of soybeans [46] or olives, implicating the potential feasibility of lipid recovery for the production of biodiesel. Herewith, we show that ecological principles can effectively be employed for the enrichment of lipid-accumulating species, enabling potential application on a broad variety of waste streams. The use of enrichment cultures decreases capital and operating costs because (semi-continuous) open cultivation techniques can be used without the need for sterilization or re-inoculation.

\section{Conclusion}

A microbial enrichment culture of lipid-storing organisms in co-existence with microorganisms that used TAG for direct formation of biomass was observed in a soybean-oil-fed reactor system. The former was represented by a lipolytic dimorphic fungus, while the latter consisted of a consortium of proteobacteria capable of utilizing either the lipid itself or its hydrolysis products, mainly as a substrate for direct growth. The maximum lipid accumulation capacity of the culture was $54 \%$ on VSS mass basis. Results were obtained from an enrichment reactor that was not optimized for maximum storage capacity, suggesting that future optimization may lead to microbial enrichment cultures with even higher lipid storage capacity.

\section{Methods}

\section{Enrichment reactor setup and operational parameters}

A SBR with a liquid volume of 21 was operated aerobically, similar to Johnson et al. An operational cycle comprised of a feed phase of $30 \mathrm{~min}$, during which TAG and nutrients were dosed, followed by a reaction phase of $11 \mathrm{~h}$. At the end of the cycle, half of the volume of the reactor was discharged, to be replaced with new feed in the next cycle, resulting in a total cycle length of $12 \mathrm{~h}$, a hydraulic retention time (HRT) and solid retention time (SRT) of $24 \mathrm{~h}$. The reactor was completely mixed $(1,000 \mathrm{rpm})$ throughout the cycle, and biofilms were structurally removed (several times per week) to ensure that the SRT equaled the hydraulic retention time. The temperature was controlled at $30^{\circ} \mathrm{C} \pm 1^{\circ} \mathrm{C}$ using a thermostat, and the $\mathrm{pH}$ was maintained at $7.0 \pm$ 0.1 by $\mathrm{pH}$ control using $1-\mathrm{M} \mathrm{NaOH}$ and $1-\mathrm{M} \mathrm{HCl}$. The gas flow into the reactor was $1.5 \mathrm{l} / \mathrm{min}$ consisting of $0.3 \mathrm{l} / \mathrm{min}$ air and $1.2 \mathrm{l} / \mathrm{min}$ recycled off-gas. The off-gas was cooled to $5^{\circ} \mathrm{C}$ by a condenser to minimize water evaporation.

\section{Medium composition}

Soybean oil (Markant merk, Beesd, Gelderland, The Netherlands) was chosen as representative model substrate for vegetable oil waste. The substrate soybean oil was analyzed by GC as described in the 'Sampling and measurement' section, and the results can be found in the 'Results' section (Table 1); the composition was similar to that of soybean oil found in literature [47]. In each cycle, $1.3 \mathrm{~g}$ (equivalent to $83 \mathrm{Cmmol}$ ) soybean oil was dosed into the reactor using a precision syringe pump. Additionally, $200 \mathrm{ml} /$ cycle nutrient solution containing $3.6 \mathrm{~g} / \mathrm{l} \mathrm{NH} \mathrm{NCl}_{4} 3.4 \mathrm{~g} / \mathrm{l} \mathrm{KH}_{2} \mathrm{PO}_{4}, 1.4 \mathrm{~g} / \mathrm{l} \mathrm{MgSO}_{4}$. $7 \mathrm{H}_{2} \mathrm{O}, 0.54 \mathrm{~g} / \mathrm{l} \mathrm{KCl}, 1.0 \mathrm{~g} / \mathrm{l}$ EDTA Titriplex III (SigmaAldrich, St. Louis, MO, USA), $0.033 \mathrm{~g} / 1 \mathrm{ZnSO}_{4} .7 \mathrm{H}_{2} \mathrm{O}$, $0.024 \mathrm{~g} / \mathrm{l} \mathrm{CoCl}_{2} \cdot 6 \mathrm{H}_{2} \mathrm{O}, 0.024 \mathrm{~g} / \mathrm{C} \mathrm{CuSO}_{4} .5 \mathrm{H}_{2} \mathrm{O}, 0.075 \mathrm{~g} / \mathrm{l}$ $\mathrm{FeSO}_{4} .7 \mathrm{H}_{2} \mathrm{O}, 0.017 \mathrm{~g} / \mathrm{l}\left(\mathrm{NH}_{4}\right)_{6} \mathrm{Mo}_{7} \mathrm{O}_{24} .4 \mathrm{H}_{2} \mathrm{O}, 0.110 \mathrm{~g} / \mathrm{l}$ $\mathrm{CaCl}_{2} \cdot \mathrm{H}_{2} \mathrm{O}$ according to Vishniac and Santer [48], and $0.050 \mathrm{~g} / \mathrm{l}$ allylthiourea (to prevent nitrification) was added together with $800 \mathrm{ml} /$ cycle of water.

\section{Sampling and measurement}

The temperature, $\mathrm{pH}$, and off-gas composition in terms of $\mathrm{CO}_{2}$ and $\mathrm{O}_{2}$ concentrations were monitored online 
using a standard sensor (Mettler Toledo, Columbus, $\mathrm{OH}$, USA) and an off-gas analyzer (NGA 2000, Rosemount Inc., Shakopee, MN, USA). In addition, samples were taken regularly at the end of the cycle to measure the TSS and the VSS concentrations according to standard methods [49] and the ammonium concentration using spectrophotometric method (LCK-348, Hach-Lange, Düsseldorf, Germany). For evaluation of the carbon balance, the organic carbon content of the organic solids was measured using a colorimetric method (LCK 381, Hach-Lange, Düsseldorf, Germany).

In order to characterize the dynamics during a cycle, samples were taken to measure the intracellular lipid concentrations, TSS and VSS production, ammonium uptake, $\mathrm{CO}_{2}$ production, and $\mathrm{O}_{2}$ consumption. Intracellular storage compounds were analyzed as described by Johnson et al. [6] except that a modified gas chromatography method was used: analysis of extracted PHA and lipids derivatives was performed using direct injection into a polyethylene glycol column (HP-INNOWax, $60 \mathrm{~m} \times$ $250 \times 0.15 \mu \mathrm{m}$ ) installed on a gas chromatograph (model 6890 N, Agilent Technologies, Santa Clara, CA, USA) equipped with a flame ionization detector. The column temperature program comprised an initial oven temperate of $100^{\circ} \mathrm{C}$, operated for $2 \mathrm{~min}$, followed by an increase to $170^{\circ} \mathrm{C}$ at a rate of $20^{\circ} \mathrm{C} / \mathrm{min}$, and a further increase to $240^{\circ} \mathrm{C}$ at a rate of $5^{\circ} \mathrm{C} / \mathrm{min}$ resulting in a total runtime of $30 \mathrm{~min}$ per sample. The temperatures of the injector and detector were $230^{\circ} \mathrm{C}$ and $250^{\circ} \mathrm{C}$, respectively. Calibration standards included PHB, PHV, hydroxyhexanoate, and hydroxypalmitate for identification of PHA, and oleic-, linoleic-, a-linolenic-, palmitic-, and stearic-acid for identification of LCFAs. For each standard, three different concentrations were used to make a calibration curve. For each GC spectrum, it was checked whether there were unknown peaks indicating missing compounds.

The lipase activity in the culture was evaluated using a colorimetric method based on the hydrolysis of an artificial lipid, 4-nitrophenyl-palmitate [36] both in untreated samples and in samples in which biomass had been removed by centrifugation for $10 \mathrm{~min}$ at 2,500 rpm. The hydrolysis product, 4-nitrophenyl was measured spectrometrically at $410 \mathrm{~nm}$.

\section{Microbial community structure}

The general microbial community composition was investigated using FISH analysis as described by Johnson et al. [6], using the EUB338 and EUK516 probe mixtures for identification of bacterial and eukaryote species, respectively [50]. Intracellular storage compounds were visualized using Nile blue A staining. The microbial diversity was further analyzed by DGGE using eukaryotic and prokaryotic primers [51]. Additionally, traditional cultivation techniques were used to separate the functional groups of microorganisms from the reactor and to isolate pure cultures. For this, serial dilutions in liquid medium containing three different substrates were used: soybean oil, the free LCFA fraction of soybean oil, and glycerol. To separate the eukaryotic community from bacteria, a mixture of kanamycine and streptomycine (100 mg/l each) was applied in a dilution series. The final positive dilutions on each substrate were plated on solid media with corresponding substrates, except that LCFA and soybean oil were first emulsified by sonication with gum arabic to allow detection of the LCFA-utilizing and lipolytic colonies [52].

\section{Fed-batch accumulation experiments}

The storage capacity of the microbial enrichment culture was assessed using a fed-batch accumulation experiment. Apart from the feeding regime, all other operational parameters were kept identical to the enrichment reactor: $\mathrm{pH}$ was maintained at $7.0 \pm 0.1$, the temperature was maintained at $30^{\circ} \mathrm{C} \pm 1^{\circ} \mathrm{C}$, and the reactor liquid was stirred with 1,000 rpm. The biomass from the enrichment reactor was used as inoculum, and the oxygen profile was used to monitor the activity in the reactor; when the oxygen consumption decreased, a new pulse of soybean oil substrate was dosed. The biomass was characterized in terms of lipid content over time.

\section{Data analysis and interpretation}

It was not possible to take a representative sample for measurement of the substrate concentration from the oilwater emulsion that formed quickly after substrate dosage. Instead the soybean oil uptake rate in the medium was estimated by assuming that all the oil had been taken up after the feast phase. This assumption was verified using microscopy to make sure that all oil had been taken up and was not absorbed to the biomass. The length of the feast phase was estimated using the off-gas data, with the feast phase characterized by relatively high oxygen consumption and $\mathrm{CO}_{2}$ production rates and the onset of the famine phase by a relatively sharp decline in both these rates (Additional file 1: Appendix A). The active biomass concentration was calculated by subtracting the amount of storage compounds from the amount of VSS in the system. Active biomass concentrations were verified by the evaluation of the ammonium uptake and nitrogen content of the biomass. Conversion yields and rates were obtained using a modified version of a model used for modeling PHA-producing feast-famine cultures [53]. The only modifications in the model were replacement of PHA storage by storage of TAG and inclusion of a fraction of the substrate that was used for direct growth. A description of the model is provided in the (Additional file 1: Appendix C). 


\section{Additional file}

Additional file 1: Appendix A: Off-gas profile. Appendix B: Carbon flow sheet. Appendix C: Model.

\section{Abbreviations}

GC: gas chromatography; HRT: hydraulic retention time; LCFA: long chain fatty acid; PHA: polyhydroxyalkanoate; SBR: sequenced batch reactor; SRT: solid retention time; TAG: triacylglyceride; TSS: total suspended solids; VFA: volatile fatty acid; VSS: volatile suspended solids.

\section{Competing interests}

The authors declare that they have no competing interests.

\section{Authors' contributions}

JT operated the reactors and performed most sampling and measurement and data analysis, and wrote the lion share of the manuscript. DS performed the main part of the characterization of the microbial composition. YJ performed the FISH analysis. RK and ML conceived of the study, participated in its design and coordination, and drafted the manuscript. All authors read and approved the final manuscript.

\section{Acknowledgements}

This research was funded by the Dutch Foundation for Technical Sciences (STW) as part of the Waste to Resource program (W2R project nr. 11605).

\section{Author details}

${ }^{1}$ Department of Biotechnology, Delft University of Technology, Julianalaan 67, 2628 BC Delft, The Netherlands. 'Winogradsky Institute of Microbiology, RAS, Leninskii avenue, 14, Leninskii avenue, 32a, Moscow 119991, Russia.

\section{Received: 10 October 2014 Accepted: 19 February 2015 Published online: 10 March 2015}

\section{References}

1. Kleerebezem R, Van Loosdrecht MCM. Mixed culture biotechnology for bioenergy production. Curr Opin Biotechnol. 2007;18:207-12.

2. Reis MAM, Serafim LS, Lemos PC, Ramos AM, Aguiar FR, Van Loosdrecht MCM. Production of polyhydroxyalkanoates by mixed microbial cultures. Bioprocess Biosyst Eng. 2003;25:377-85.

3. Agler MT, Wrenn BA, Zinder SH, Angenent LT. Waste to bioproduct conversion with undefined mixed cultures: the carboxylate platform. Trends Biotechnol. 2011:29:70-8.

4. Spirito CM, Richter H, Rabaey K, Stams AJM, Angenent LT. Chain elongation in anaerobic reactor microbiomes to recover resources from waste. Curr Opin Biotechnol. 2014;27:115-22.

5. Jiang $Y$, Marang $L$, Kleerebezem R, Muyzer G, Van Loosdrecht MCM. Polyhydroxybutyrate production from lactate using a mixed microbial culture. Biotechnol Bioeng. 2011;108:2022-35.

6. Johnson K, Jiang Y, Kleerebezem R, Muyzer G, Van Loosdrecht MCM. Enrichment of a mixed bacterial culture with a high polyhydroxyalkanoate storage capacity. Biomacromolecules. 2009;10:670-6.

7. Anterrieu S, Quadri L, Geurkink B, Dinkla I, Bengtsson S, Arcos-Hernandez M, et al. Integration of biopolymer production with process water treatment at a sugar factory. N Biotechnol. 2014;31:308-23.

8. Jiang Y, Marang L, Tamis J, Van Loosdrecht M, Dijkman H, Kleerebezem R. Waste to resource: converting paper mill wastewater to bioplastic. Water Res. 2012;46:5517-30.

9. Liu H-Y, Hall PV, Darby JL, Coats ER, Green PG, Thompson DE, et al. Production of polyhydroxyalkanoate during treatment of tomato cannery wastewater. Water Environ Res. 2008:80:367-72

10. Carta F, Beun JJ, Van Loosdrecht MCM, Heijnen JJ. Simultaneous storage and degradation of PHB and glycogen in activated sludge cultures. Water Res. 2001;35:2693-701

11. Karahan Ö, Van Loosdrecht MCM, Orhon D. Modeling the utilization of starch by activated sludge for simultaneous substrate storage and microbial growth. Biotechnol Bioeng. 2006:94:43-53.

12. Moralejo-Gárate $H$, Mar'atusalihat E, Kleerebezem R, Loosdrecht MM. Microbial community engineering for biopolymer production from glycerol. Appl Microbiol Biotechnol. 2011;92:631-9.
13. Albuquerque MGE, Martino V, Pollet E, Avérous L, Reis MAM. Mixed culture polyhydroxyalkanoate (PHA) production from volatile fatty acid (VFA)-rich streams: effect of substrate composition and feeding regime on PHA productivity, composition and properties. J Biotechnol. 2011;151:66-76.

14. Jiang Y, Hebly M, Kleerebezem R, Muyzer G, Van Loosdrecht M. Metabolic modeling of mixed substrate uptake for polyhydroxyalkanoate (PHA) production. Water Res. 2011;45:1309-21.

15. Marang $L$, Jiang $Y$, van Loosdrecht $M$, Kleerebezem R. Impact of non-storing biomass on PHA production: an enrichment culture on acetate and methanol. International journal of biological macromolecules. 2014;71:74-80.

16. Igwe J, Onyegbado C. A review of palm oil mill effluent (POME) water treatment. Global J Environ Res. 2007;1:54-62.

17. FAO: FAOSTAT. FAOSTAT. Food \& Agriculture Organization of the United Nations; 2014

18. Borja R, Banks C. Anaerobic digestion of palm oil mill effluent using an up-flow anaerobic sludge blanket reactor. Biomass Bioenergy. 1994;6:381-9.

19. Muller EE, Sheik AR, Wilmes P. Lipid-based biofuel production from wastewater. Curr Opin Biotechnol. 2014;30:9-16.

20. Mondala A, Liang K, Toghiani $H$, Hernandez R, French T Biodiesel production by in situ transesterification of municipal primary and secondary sludges. Bioresour Technol. 2009;100:1203-10.

21. Wakelin NG, Forster CF. An investigation into microbial removal of fats, oils and greases. Bioresour Technol. 1997:59:37-43.

22. Eggink $G$, van der Wal H, Huijberts GN, De Waard P. Oleic acid as a substrate for poly-3-hydroxyalkanoate formation in Alcaligenes eutrophus and Pseudomonas putida. Ind Crop Prod. 1992;1:157-63.

23. Lageveen RG, Huisman GW, Preusting H, Ketelaar P, Eggink G, Witholt B. Formation of polyesters by Pseudomonas oleovorans: effect of substrates on formation and composition of poly-(R)-3-hydroxyalkanoates and poly-(R)-3-hydroxyalkenoates. Appl Environ Microbiol. 1988;54:2924-32

24. Lee SH, Oh DH, Ahn WS, Lee Y, Choi J, Lee SY. Production of poly (3-hydroxybutyrate-co-3-hydroxyhexanoate) by high-cell-density cultivation of Aeromonas hydrophila. Biotechnol Bioeng. 2000;67:240-4.

25. Ng K-S, Ooi W-Y, Goh L-K, Shenbagarathai R, Sudesh K. Evaluation of jatropha oil to produce poly (3-hydroxybutyrate) by Cupriavidus necator H16. Polym Degrad Stab. 2010:95:1365-9.

26. Liang $\mathrm{M}-\mathrm{H}$, Jiang J-G. Advancing oleaginous microorganisms to produce lipid via metabolic engineering technology. Prog Lipid Res. 2013;52:395-408.

27. Zhu L, Zong M, Wu H. Efficient lipid production with Trichosporon fermentans and its use for biodiesel preparation. Bioresour Technol. 2008;99:7881-5.

28. Alvarez HA, Steinbüchel AS. Triacylglycerols in prokaryotic microorganisms. Appl Microbiol Biotechnol. 2002;60:367-76.

29. Slijkhuis HVG, Johan W, Kylstra, Dirk J. Microthrix parvicella, a filamentous bacterium from activated sludge: growth on Tween 80 as carbon and energy source. J Gen Microbiol. 1984;130:2035-42.

30. Ling J, Nip S, Shim H. Enhancement of lipid productivity of Rhodosporidium toruloides in distillery wastewater by increasing cell density. Bioresource technology. 2013;146:301-9.

31. W-f P, Huang C Chen X-f, Xiong L, Chen Y, Ma L-I. Microbial conversion of wastewater from butanol fermentation to microbial oil by oleaginous yeast Trichosporon dermatis. Renewable Energy. 2013:55:31-4.

32. Santamauro F, Whiffin FM, Scott RJ, Chuck CJ. Low-cost lipid production by an oleaginous yeast cultured in non-sterile conditions using model waste resources. Biotechnology for biofuels. 2014;7:34.

33. Magan N. Fungi in extreme environments. In: Kubicek CP, Esser K Druzhinina IS, editors. Environmental and microbial relationships. New York: Springer; 2007. p. 85

34. Mooij PR, Stouten GR, Van Loosdrecht MCM, Kleerebezem R. Ecology-based selective environments as solution to contamination in microalgal cultivation. Curr Opin Biotechnol. 2015:33:46-51.

35. Andreasen K, Nielsen PH. Growth of Microthrix parvicella in nutrient removal activated sludge plants: studies of in situ physiology. Water Res. 2000;34:1559-69.

36. Vorderwulbecke T, Kieslich K, Erdmann H. Comparison of lipases by different assays. Enzyme Microb Technol. 1992;14:631-9.

37. Holdsworth JE, Ratledge C. Lipid turnover in oleaginous yeasts. J Gen Microbiol. 1988;134:339-46.

38. Arenskötter $M$, Bröker $D$, Steinbüchel $A$. Biology of the metabolically diverse genus Gordonia. Appl Environ Microbiol. 2004;70:3195-204.

39. Juni E. Genetics and physiology of Acinetobacter. Annu Rev Microbio. 1978:32:349-71. 
40. Chipasa K, Mędrzycka K. Behavior of lipids in biological wastewater treatment processes. J Ind Microbiol Biotechnol. 2006;33:635-45.

41. Gouda MK, Omar SH, Aouad LM. Single cell oil production by Gordonia sp. DG using agro-industrial wastes. World J Microbiol Biotechnol. 2008;24:1703-11

42. Jiang Y, Marang L, Kleerebezem R, Muyzer G, Van Loosdrecht MCM. Effect of temperature and cycle length on microbial competition in PHB-producing sequencing batch reactor. International Society for Microbial Ecology. 2011;5:896-907.

43. Sigurgísladóttir S, Konráòsdóttir M, Jónsson Á, Kristjánsson J, Matthiasson E. Lipase activity of thermophilic bacteria from icelandic hot springs. Biotechnol Lett. 1993;15:361-6.

44. Cirne DG, Paloumet X, Björnsson L, Alves MM, Mattiasson B. Anaerobic digestion of lipid-rich waste - effects of lipid concentration. Renew Energy. 2007;32:965-75

45. Johnson K, Kleerebezem R, Van Loosdrecht M. Influence of ammonium on the accumulation of polyhydroxybutyrate (PHB) in aerobic open mixed cultures. J Biotechnol. 2010;147:73-9.

46. FAO. Technology of production of edible flours and protein products from soybeans. FAO agricultural services bulletin no 97. 1992.

47. Babayan VK. Medium chain triglycerides and structured lipids. Lipids. 1987:22:417-20

48. Vishniac W, Santer M. Thiobacilli. Bacteriol Rev. 1957;21:195-213.

49. Clesceri LS, Greenberg AE, Eaton AD. Standard methods for the examination of water and wastewater. 20th ed. USA: American Public Health Association; 1999.

50. Alm EW, Oerther DB, Larsen N, Stahl DA, Raskin L. The oligonucleotide probe database. Appl Environ Microbiol. 1996;62:3557.

51. Muyzer G. DGGE/TGGE a method for identifying genes from natural ecosystems. Curr Opin Microbiol. 1999;2:317-22.

52. Sorokin DY, Jones BE. Improved method for direct screening of true lipase-producing microorganisms with particular emphasis on alkaline conditions. Microbiology. 2009;78:125-30.

53. Tamis J, Marang L, Jiang Y, Van Loosdrecht MC, Kleerebezem R. Modeling PHA-producing microbial enrichment cultures - towards a generalized model with predictive power. N Biotechnol. 2014:31:324-34.

\section{Submit your next manuscript to BioMed Central and take full advantage of:}

- Convenient online submission

- Thorough peer review

- No space constraints or color figure charges

- Immediate publication on acceptance

- Inclusion in PubMed, CAS, Scopus and Google Scholar

- Research which is freely available for redistribution 